\title{
DISPERSED TWO-PHASE FLOW IN A GAS-LIQUID CYLINDRICAL CYCLONE SEPARATOR
}

\author{
CARSTEN MEHRING ${ }^{1,2}$ \\ ${ }^{1}$ Aerospace Group, Central Engineering, Parker Hannifin Corporation, USA. \\ ${ }^{2}$ Department of Mechanical \& Aerospace Engineering, University of California, USA.
}

\begin{abstract}
The performance of a gas-liquid cylindrical cyclone separator for the separation of air bubbles from hydraulic fluid has been analyzed numerically using the commercial computational fluid dynamics flow solver CFX. Two-phase flow behavior is modeled based on an Eulerian-Eulerian approach, representing both liquid and dispersed gas phase as interpenetrating media with interphase momentum transfer captured based on existing bubble drag models. Only a single bubble size is considered for the dispersed phase and bubble coalescence is ignored. At the tangential inlet, a homogeneous gasliquid mixture is assumed with specified mass flow and air/liquid volume fractions. Pressure conditions were imposed at both the upper gas outlet and the lower liquid outlet boundaries. Effect of changes in turbulence model and bubble drag model on analysis predictions was analyzed for selected operating conditions, that is, bubble sizes, air/liquid volume fractions and flow rates. Separation efficiency was high only for larger bubble sizes $(100 \mathrm{~mm})$ and high flow rates $(250 \mathrm{~L} / \mathrm{min})$. For bubble sizes below 35 $\mathrm{mm}$, the cyclone was ineffective. At high flow rates, cyclone performance suffers due to liquid carry over in the form of a swirling liquid wall film carried with the gas (bubble) phase through the top outlet. The Explicit Algebraic Reynolds Stress Turbulence Model together with the Grace Drag Model was found to be effective in exploring changes to the cyclone geometry. Here, improvements in separation efficiency were only achieved with significantly increased tangential velocities as a consequences of a reduced inlet port cross section. Predicted bubble size separation limits as a function of Reynolds number and axial-to-tangential velocity ratios were found to compare favorably with existing literature. Keywords: bubbly flow, hydrocyclone, modeling, phase separation.
\end{abstract}

\section{INTRODUCTION}

Hydrocyclones, also referred to as liquid or hydraulic cyclones, are used in a broad range of processing industries (mineral, chemical, food and pharmaceutical) for the purpose of separating materials of different densities from a liquid primary phase or as particle size classifiers in the case of solid particulates dispersed within the primary phase. Their use has increased in popularity since the 1950s due to their simple and compact design and low operational and manufacturing costs compared to other separation devices and methods [1,2]. More recently, they have also found attention in context of fluid deaeration devices in hydraulic vehicle systems and as phase separation devices under microgravity conditions [3,4]. A considerable amount of literature has been presented on the general topic, starting with experimental investigations, empirical correlations and algebraic models to estimate velocities, pressure drop, collection efficiencies and overall performance and as function of cyclone geometry [5-8]. For a more recent review [9,10]. With advances in numerical methods and computer hardware, computational fluid dynamics analysis has been deployed since the 1990s to improve the understanding of the complex phenomena in cyclones, characterized by non-isotropic turbulence and

This paper is part of the Proceedings of the $8^{\text {th }}$ International Conference on Computational and Experimental Methods in Multiphase and Complex Flow (Multiphase Flow 2015)

www.witconferences.com
} 

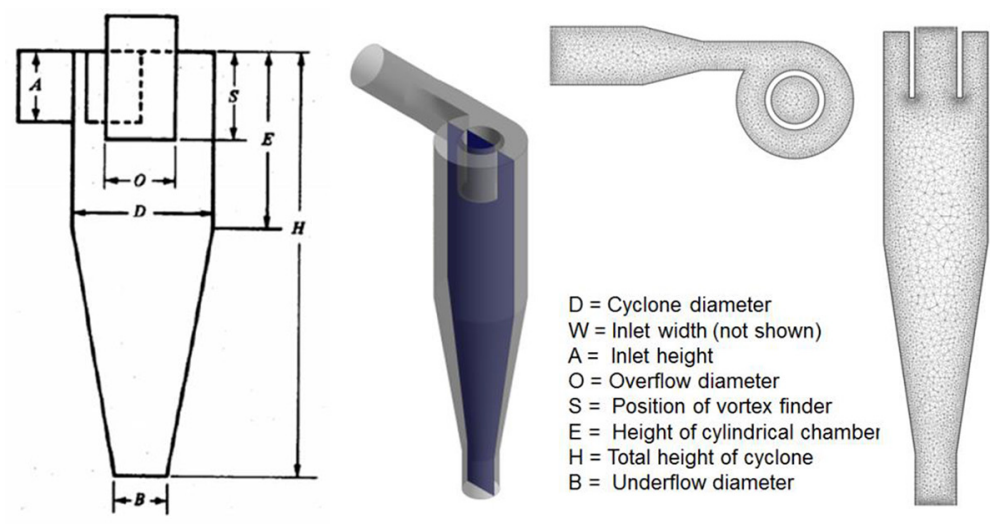

Figure 1: Geometry of cylindrical hydrocyclone. Parametric model allows for variation in radii of over- and underflow, vertical extend of vortex finder (S) and length of cylindrical cyclone section (E) while keeping overall cyclone length $(\mathrm{H})$ constant.

complex multi-phase flow behavior. The earliest analyses in this context have been reported by Boysan [11], Pericleous and Rhodes [12] and Hsieh and Rajamani [13,14]. With deepened understanding and increases in computational resources, analysis models have expanded in complexity to include higher order discretization schemes and increasingly complex multi-phase models paired with anisotropic turbulence models such as various different Reynolds stress models (RSM) in RANS (Reynolds-averaged Navier-Stokes) simulations or unsteady RANS and large-eddy simulation (LES). For a more detailed discussion [15].

The aim of the present study was to explore the performance characteristics of a given hydrocyclone design and to identify a numerical approach suitable for use in a design environment, that is, broader design space exploration. The considered cylindrical cyclone see Fig. 1 operates with Tellus Oil 32 as its primary phase and is aimed at separating a secondary phase of air bubbles finely dispersed within the primary phase. The prescribed study was carried out by deploying the commercial flow solver ANSYS CFX (v.15.0). Various available turbulence models were evaluated at selected operating conditions and two different drag models were considered for the inter-phase momentum transfer, Finally, a degassing boundary condition available in ANSYS CFX has also been evaluated for the overflow exit. Based on the findings from these analyses, the BSL EARS (Baseline Explicit Algebraic Reynolds Stress) Model and the Grace Drag Model were chosen for a DOE study to improve cyclone efficiency. Details of that study are not presented here, but were in agreement with previously published literature on the subject [16].

\section{MODEL DESCRIPTION}

\subsection{Geometry and mesh}

The geometry of the cylindrical hydrocyclone considered within this paper is shown in Fig. 1. A parametric model was built allowing for modifications of vortex finder (vertical position), radius of overflow exit, height of the cyclone's cylindrical section and radius of underflow exit. For all analyses, a combined tetrahedral/prism mesh was used. To assess mesh sensitivity, initial analyses were carried out for both medium and fine mesh sizes, with 611,000 and 1.8 million nodes, respectively. To keep computational times and resource allocation manageable, the medium size mesh was chosen for the study after the initial mesh sensitivity assessment. 
All analyses have been carried out on an HPC platform with computational core allocation ranging from 8 to 32 and wall-clock run-times between several hours and 1 day.

\subsection{Boundary conditions}

Mass flow inlet boundary conditions were specified at the tangential inlet of the hydrocyclone together with specification of the phase volume fractions (loading). The cyclone underflow and overflow exit boundaries were specified as outflow boundaries with static pressures specified at those locations. For a few select cases, the overflow exit boundary was specified as degassing boundary allowing for air bubbles to escape through the overflow while preventing the liquid phase from exiting. All wetted solid walls were assumed to be smooth with $U_{\text {wall }}=0$ (non-slip condition).

\subsection{Analysis model}

Fluid properties for Tellus Oil 32 and air were assumed to be constant and have been evaluated at $40^{\circ} \mathrm{C}$ and $1 \mathrm{~atm}$, despite the fact that pressures canvary considerably across the cyclone effectively resulting in a change of bubble size. The oil was assumed to be free of dissolved air and heat and mass transfer across the bubble interfaces was assumed to be zero. Clearly, only momentum transfer was considered for the present analysis, and a conservation equation for energy was not solved.

\subsubsection{Transport equations/two-phase flow model}

The inhomogeneous Eulerian-Eulerian multiphase flow model within CFX was used to model the two-phase oil/air bubble flow through the cyclone with inter-phase momentum transfer captured by its dispersed-phase model. For the present analysis, only particle drag and buoyancy force were considered. Non-drag forces due to lift, virtual mass, wall lubrication and turbulent dispersion were neglected.

Assuming constant temperature and in the absence of mass transfer, the mass conservation equation for phase $k$ in a mixture of fluids can be written as

$$
\frac{\partial}{\partial t}\left(a_{k} \rho_{k}\right)+\frac{\partial}{\partial x_{i}}\left(a_{k} \rho_{k} U_{k, i}\right)=0
$$

with $\alpha_{k}, \rho_{k}$ and $U_{\mathbf{k}, \mathbf{i}}$ denoting volumetric fraction, density and mean velocity components of phase $k$. Similarly, the momentum balance equation for phase $k$ is given by

$$
\begin{gathered}
\frac{\partial}{\partial t}\left(\alpha_{k} \rho_{k} U_{k, i}\right)+\frac{\partial}{\partial x_{j}}\left(a_{k} \rho_{k} U_{k, i} U_{k, i}\right) \\
=-a_{k} \frac{\partial P}{\partial x_{i}}+I_{k, i}+a_{k} \rho_{k} g_{i}+\frac{\partial}{\partial x_{j}} S_{k, i, j}^{\prime}
\end{gathered}
$$

where $P$ is the mean pressure (i.e. same pressure field for all phases), $I_{k, i}$ is the average interphase momentum transfer, $g_{i}$ denotes component $i$ of the gravitational acceleration vector and $S_{k, i j}$ the effective stress tensor. For the case of particulate or bubbly flow, the momentum transfer rate from the continuous liquid phase to the particulate/bubble phase can be modeled using an estimate of the drag force between the phases. Referring $k=c$ to the continuous liquid phase and $k=d$ to the discrete bubble phase, we have 


$$
I_{c, i}=-I_{d, i}=\frac{\alpha_{d} \rho_{d}}{\tau_{c, d}}\left(U_{d, i}-U_{c, i}\right)
$$

with

$$
\frac{1}{\tau_{c, d}}=\frac{3}{4} \frac{\rho_{c} C_{D}}{p_{d} d} \mid \overrightarrow{U_{d}}-\vec{U}_{c}
$$

where $\tau_{c, d}$ describes a particle relaxation time and $C_{D}$ the drag coefficient for the monodisperse particulate phase of spherical bubbles with diameter $d$.

The components of the effective stress tensor for phase $k$ are given by

$$
S_{k, i, j}^{\prime}=\alpha_{k} \mu\left[\left(\frac{\partial U_{k, i}}{\partial x_{j}}+\frac{\partial U_{k, j}}{\partial x_{i}}\right)-\frac{2}{3} \operatorname{div}\left(\vec{U}_{k}\right) \delta_{i j}\right]-\alpha_{k} \rho_{k} \overline{u_{k, i}^{\prime} u_{k, j}^{\prime}}
$$

where the first terms reflect molecular stresses and the last term denotes components of the Reynolds Stress tensor as a consequence of turbulent velocity fluctuations $u_{k, i}^{\prime}$ in phase $k$. In ANSYS CFX, interphase transfer of turbulent quantities between phases such as turbulent kinetic energy and its dissipation rate are neglected.

\subsubsection{Turbulence and drag models}

Various turbulence models available within the employed commercial flow solver were evaluated, that is, the Baseline Explicit Algebraic Reynolds Stress Model (BSL EARSM), the Shear Stress Transport Model with Curvature Correction (SST-CC) in both steady and unsteady modes, the Baseline Reynolds Stress Model (BSL RSM) and the Speziale-SarkarGatski Reynolds Stress Model (SSG RSM). In addition, two different drag models were considered for the interphase momentum transfer, that is, the Schiller-Naumann Drag Model and the Grace Drag Model. For a detailed description of these models, the reader is referred to the ANSYS CFX Solver Theory Guide (Release 15.0).

Since the BSL EARSM Model and the Grace Drag Model were later used for design studies, these models are described in more detail.

Within the BSL EARSM Model, Reynolds stresses are being related to the mean strain rate and vorticity tensors via explicit relations, that is,

$$
\overline{u_{i}^{\prime} u_{j}^{\prime}}=k\left(A_{i j}+\frac{2}{3} \delta_{i j}\right) ; A_{i j}=f c t n\left(\psi_{i j}, \Omega_{i j}\right)
$$

where a subscript indicating the fluid phase has been omitted, $\delta_{i j}$ denotes the Kronecker delta, $k$ denotes turbulent kinetic energy and the components of the anisotropy tensor $A_{i j}$ are complex function of the non-dimensional strain rate tensor $\Psi_{i, j}$ and vorticity tensor $\Omega_{i, j}$ components, that is,

$$
\psi i j=\frac{1}{2} \tau\left(\frac{\partial U i}{\partial x j}+\frac{\partial U j}{\partial x i}\right) ; \quad \Omega i j=\frac{1}{2} \tau\left(\frac{\partial U i}{\partial x j}-\frac{\partial U j}{\partial x i}\right)
$$


with time-scale constant $\tau=k / \varepsilon=1 /\left(C_{\mu} \omega\right)$, where $C_{\mu}=0.09, \varepsilon$ is the dissipation rate of $k$ and $\omega$ the turbulence eddy frequency. The BSL EARSM model deploys the Wilcox $k-\omega$ model near solid surfaces and the $k-\varepsilon$ model away from the wall [17]. In the present application, the BSL EARSM model only applies to the continuous phase. A zero equation or mixing length turbulence model is used for the dispersed (bubble) phase with the dispersed phase eddy viscosity, $\mu_{t, d}$ being proportional to that of the continuous phase $\mu_{t, c}$, that is,

$$
\mu_{t, d}=\frac{\rho_{d}}{\rho_{c}} \frac{1}{\sigma} \mu_{t c}
$$

where $\sigma$ denotes the turbulent Prandtl number.

Unlike the Schiller-Naumann model which considers spherical bubbles only; the Grace Drag model in addition allows for bubble distortion by considering two distortion regimes, that is, the ellipsoidal and the spherical cap regimes. A simple min/max decision logic selects the correct bubble regime. Correlations for each regime are based on isolated bubbles giving regime-specific drag coefficients $C_{D, \infty}$. The model corrects for bubble/bubble interaction at high bubble volume fractions by using as simple power law, that is,

$$
C_{d}=a_{c}^{p} C_{d, \infty}
$$

with the volume fraction correction exponent $p=0$ (dilute bubble phase), -0.5 (small bubbles) and 2-4 (larger bubbles).

\section{RESULTS}

Table 1 summarizes the analysis cases discussed within this paper together with information on operating conditions, that is, air/liquid loading, bubble size, mass inflow and boundary conditions. The original naming convention for the various cases has been retained. Some line-items have been removed since not all cases are presented here.

\subsection{Effect of droplet drag model, bubble size and flow rate}

The effect of different interphase drag models and changes in boundary conditions was first studied for low mass inflow rate and high air loading with small bubble size, that is, $50 \mathrm{~L} / \mathrm{min}$ at $25 \%$ air volume fraction with $35 \mu \mathrm{m}$ bubbles. Cases 3 and 15 of Table 1 correspond to this configuration with the Schiller Naumann and grace drag models and an overflow static pressure of $P_{s, o}=1.2 \mathrm{psig}\left(P_{s, u}=0.696 \mathrm{psig}\right)$. Figure 2 shows contour plots for these cases illustrating that at the small bubble size (and in the absence of a volume correction in case of the Grace Drag Model); results are virtually identical with no phase separation taking place.

Comparison of Cases 20 and 21 in Fig. 3 illustrates that with an increase in bubble size from 35 to $100 \mu \mathrm{m}$ and volumetric flow increase from 50 to $250 \mathrm{~L} / \mathrm{min}$ while keeping boundary pressures and volumetric air loading constant, the given cyclone geometry becomes reasonably effective going from virtually no phase separation (Case 20) to delivering approximately $86 \%$ of the inflow air through the overflow outlet and $73 \%$ of the inflow oil through the underflow exit. (Here, $P_{s, o}=P_{s, u}=0$ psig was imposed, i.e. both outlets are open to atmosphere.) The corresponding average air volume fraction at the overflow outlet is $52 \%$ and at the underflow outlet $7 \%$. Note that, while a considerable amount of liquid/oil is still passing through the 
Table 1: Selected analysis cases considered within the present study.

\begin{tabular}{|c|c|c|c|c|c|c|c|c|c|c|}
\hline \multirow{3}{*}{ Cases } & \multirow{3}{*}{$\begin{array}{l}\text { Flow } \\
\text { Rate } \\
(1 / \mathrm{min})\end{array}$} & \multirow{2}{*}{\multicolumn{2}{|c|}{$\begin{array}{c}\text { Loading } \\
\text { Percentage } \\
\%\end{array}$}} & \multirow{3}{*}{$\begin{array}{l}\text { Bubble } \\
\text { Dia } \\
\text { micron }\end{array}$} & \multirow{2}{*}{\multicolumn{2}{|c|}{$\begin{array}{c}\text { Inlet BC } \\
\text { Mass Flow Rate } \\
(\mathrm{kg} / \mathrm{s})\end{array}$}} & \multirow{2}{*}{\multicolumn{2}{|c|}{$\begin{array}{c}\text { Outlet BC } \\
\begin{array}{c}\text { Pressure BC } \\
\text { (psig) }\end{array}\end{array}$}} & \multirow{3}{*}{$\begin{array}{l}\text { Drag } \\
\text { Model }\end{array}$} & \multirow{3}{*}{$\begin{array}{c}\text { Turbulence } \\
\text { Model }\end{array}$} \\
\hline & & & & & & & & & & \\
\hline & & Gas & Oil & & Gas & Oil & Gas & Oil & & \\
\hline 1 & 50 & 25 & 75 & 35 & $2.35 \mathrm{E}-04$ & $5.47 \mathrm{E}-01$ & $\begin{array}{l}\text { Deg. } \\
\text { Cond. }\end{array}$ & 0.696 & $\mathrm{G}$ & BSL EARSM \\
\hline 2 & 50 & 25 & 75 & 35 & $2.35 \mathrm{E}-04$ & $5.47 \mathrm{E}-01$ & $\begin{array}{l}\text { Deg. } \\
\text { Cond. }\end{array}$ & 0.696 & S-N & BSL EARSM \\
\hline 3 & 50 & 25 & 75 & 35 & $2.35 \mathrm{E}-04$ & $5.47 \mathrm{E}-01$ & 1.200 & 0.696 & S-N & BSL EARSM \\
\hline $3 a$ & 50 & 25 & 75 & 35 & $2.35 \mathrm{E}-04$ & $5.47 \mathrm{E}-01$ & 1.000 & 0.696 & S-N & BSL EARSM \\
\hline 4 & 50 & 25 & 75 & 35 & 2.35E-04 & $5.47 \mathrm{E}-01$ & 1.000 & 0.696 & G & BSL EARSM \\
\hline 7 & 50 & 25 & 75 & 35 & $2.35 \mathrm{E}-04$ & $5.47 \mathrm{E}-01$ & $\begin{array}{l}\text { Deg. } \\
\text { Cond. }\end{array}$ & 0.696 & G & BSL EARSM \\
\hline 13 & 50 & 5 & 95 & 35 & 4.70E-05 & $6.93 \mathrm{E}-01$ & 1.200 & 0.696 & G & BSL EARSM \\
\hline 15 & 50 & 25 & 75 & 35 & $2.35 \mathrm{E}-04$ & $5.47 \mathrm{E}-01$ & 1.200 & 0.696 & G & BSL EARSM \\
\hline 20 & 50 & 25 & 75 & 35 & $2.35 \mathrm{E}-04$ & $5.47 \mathrm{E}-01$ & 0.000 & 0.000 & G & BSL EARSM \\
\hline 21 & 250 & 25 & 75 & 100 & $1.17 \mathrm{E}-03$ & $2.73 \mathrm{E}+00$ & 0.000 & 0.000 & G & BSL EARSM \\
\hline $21 / 1$ & & & & & & & & & & SST-CC \\
\hline $21 / 2$ & & & & & & & & & & $\begin{array}{l}\text { SST-CC } \\
\text { (trans) }\end{array}$ \\
\hline $21 / 3$ & & & & & & & & & & SSG RSM \\
\hline $21 \mathrm{a}$ & 250 & 25 & 75 & 250 & $1.17 \mathrm{E}-03$ & $2.73 \mathrm{E}+00$ & 0.000 & 0.000 & G & BSL EARSM \\
\hline 22 & 500 & 25 & 75 & 100 & $2.35 \mathrm{E}-03$ & $5.47 \mathrm{E}+00$ & 0.000 & 0.000 & G & BSL EARSM \\
\hline 25 & 50 & 25 & 75 & 100 & $2.35 \mathrm{E}-04$ & $5.47 \mathrm{E}-01$ & 0.000 & 0.000 & G & BSL EARSM \\
\hline $25 / 1$ & & & & & & & & & & $\begin{array}{l}\text { SST-CC } \\
\text { (trans) }\end{array}$ \\
\hline $25 / 2$ & & & & & & & & & & SSG RSM \\
\hline 26 & 250 & 25 & 75 & 100 & $1.17 \mathrm{E}-03$ & $2.73 E+00$ & 0.000 & 0.000 & S-N & BSL EARSM \\
\hline 27 & 250 & 5 & 95 & 35 & $2.35 \mathrm{E}-04$ & $3.46 \mathrm{E}+00$ & 0.000 & 0.000 & G & BSL EARSM \\
\hline $27 / 1$ & & & & & & & & & & BSL RSM \\
\hline $27 / 2$ & & & & & & & & & & SST-CC \\
\hline $27 / 3$ & & & & & & & & & & $\begin{array}{l}\text { SST-CC } \\
\text { (trans) }\end{array}$ \\
\hline $27 / 4$ & & & & & & & & & & SSG RSM \\
\hline 28 & 250 & 25 & 75 & 100 & $1.17 \mathrm{E}-03$ & $2.73 \mathrm{E}+00$ & 0.000 & 0.000 & $\begin{array}{c}\mathrm{G} \\
(\mathrm{vc}=4)\end{array}$ & BSL EARSM \\
\hline
\end{tabular}

S-N, Schiller-Naumann drag model; G, Grace drag model. 

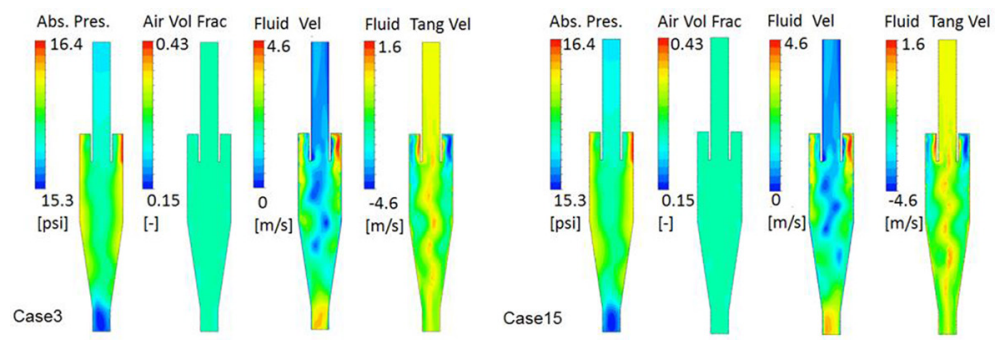

Figure 2: Contour plots of absolute static pressure, air volume fraction, fluid absolute velocity and fluid circumferential velocity for Cases 3 and 15.
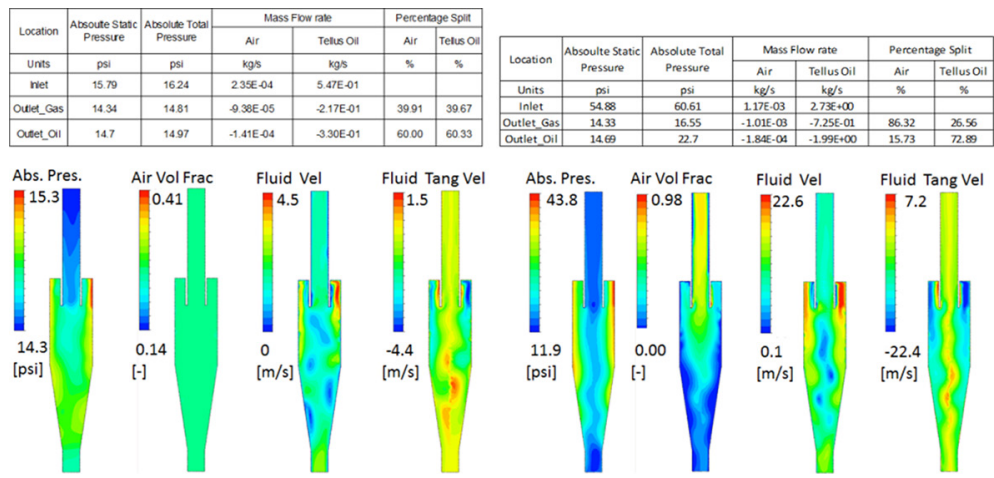

Figure 3: Contour plots of absolute static pressure, air volume fraction, fluid absolute velocity and fluid circumferential velocity for Case 20 (left) and Case 21 (right).

overflow, the liquid phase exits in the form of an annular film, providing a good opportunity to achieve additional phase separation. As the bubble size is further increased from 100 to 250 $\mu \mathrm{m}$ or as the flow rate is increased from 250 to $500 \mathrm{~L} / \mathrm{min}$, nearly $100 \%$ of air-phase separation is achieved from the underflow stream, even though fluid carryover at the overflow outlet has increased from $26 \%$ to around $39 \%$ (see Fig. 4 in this context). Again the same wall film is formed as for Case 21, allowing a simple scavenging of that film to further improve efficiency.

At the larger bubble sizes, differences between analysis results for Grace Drag Model and Schiller-Naumann Drag Model increase, with phase separation being more effective in cases where the Grace Drag Model was deployed. Further increase in separation efficiency is found when the volume fraction correction coefficient in the Grace Model is increased from its nominal value 2 to 4 . This behavior is demonstrated by comparing the results of Case 21 (with Grace Drag Model, see Fig. 3) to Case 26 (with Schiller Naumann Drag Model) and Case 28 (Grace Drag Model with volume correction coefficient increased from 2 to 4) illustrated in Fig. 5.

Careful drag model selection is needed if quantitative analysis predictions are to be made. The latter might also need to include an extension of the deployed particle model, which considers a realistic size distribution of the expected bubbly flow as well as bubble break-up and coalescence; the former especially due to the high shear encountered in the present flow and the latter due to bubble number density increase near the cyclone centerline and in the overflow exit. 

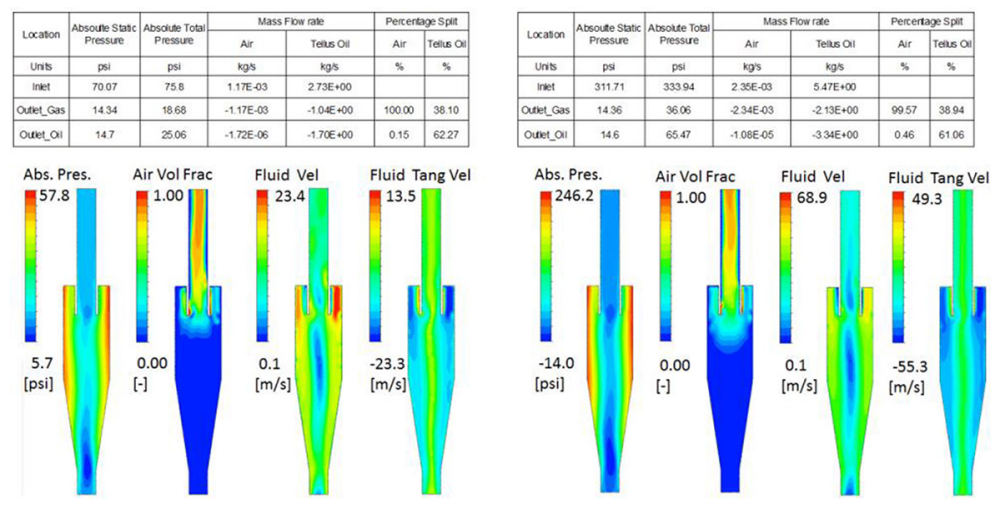

Figure 4: Contour plots of absolute static pressure, air volume fraction, fluid absolute velocity and fluid circumferential velocity for Case 21a (left) and Case 22 (right).
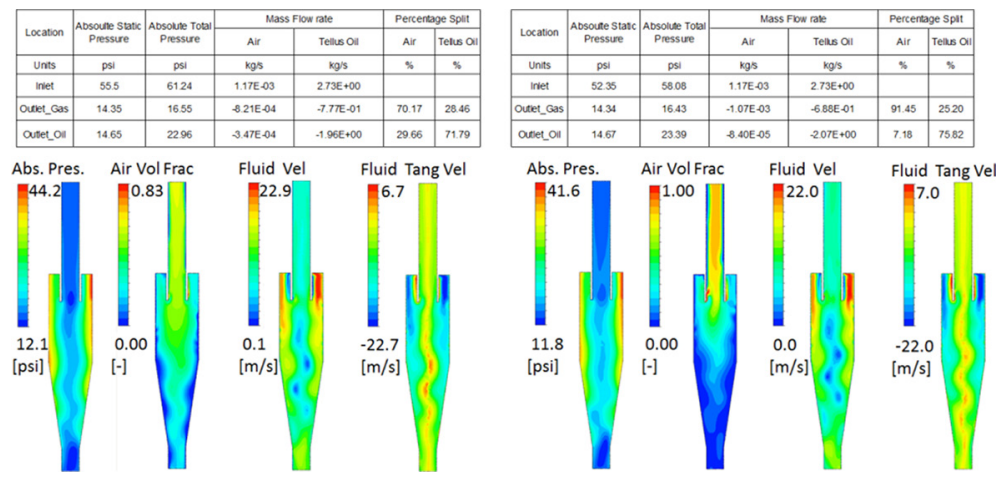

Figure 5: Contour plots of absolute static pressure, air volume fraction, fluid absolute velocity and fluid circumferential velocity for Case 26 (left) and Case 28 (right).

\subsection{Degassing condition at overflow boundary}

The construct of a degassing boundary condition available in ANSYS CFX was evaluated at the cyclone overflow boundary. This boundary condition mimics a free surface of the primary/continuous liquid phase, allowing for raising air bubbles to pass through that boundary while the boundary is impermeable to the continuous phase itself.

Comparison of Case 2 with Case 7 and Case 3 with Case 15 (see Figs 6 and 2) illustrates how the prescribed degassing boundary condition changes analysis results in comparison to a corresponding pressure boundary condition and as function of the employed particle drag model. Note that for Cases 3 and 15, the pressure value was set to the static pressure predicted at that location for cases using the degassing boundary condition (i.e. $P_{s, o}=1.2 \mathrm{psig}$ ). The degassing condition renders the overflow tube nearly stagnant and with only little air flow passing through that exit boundary. For bubble rise determined by the Schiller-Naumann Drag Model, no reverse air flow is observed at the exit boundary. However, for the Grace Model, bubble rise and exit flux are enhanced and cannot be maintained resulting in reverse air flow, which leads to the formation of a large air core near the axis of the cylindrical portion 

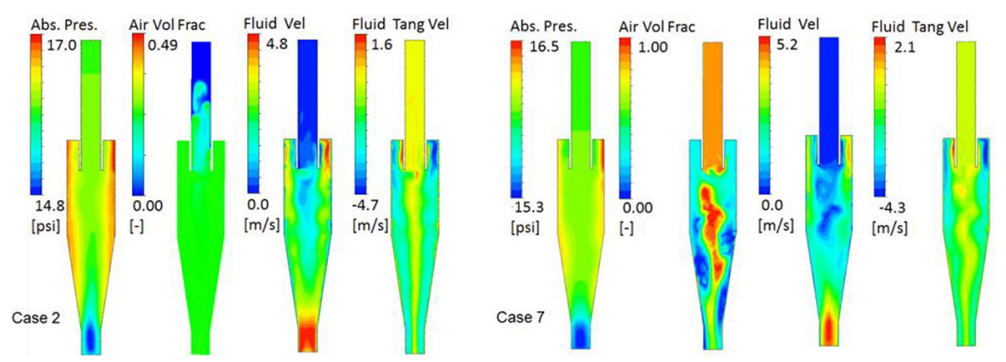

Figure 6: Contour plots of absolute pressure, air volume fraction, fluid velocity and fluid circumferential velocity for Case 2 (left) and Case 7 (right).

of the cyclone. In both cases, air mass flow in the overflow is small and nearly all air volume entering the system is passed through the underflow exit. It is interesting to note that for the Schiller-Naumann Drag Model (i.e. no airflow return from the degassing boundary), the vortex core appears stabilized close to the cyclone centerline. Clearly, as with all boundary conditions, the degassing boundary condition should be deployed only when the user is confident that a liquid-free surface would exits at that location within the overall system. Consequently, within the present study pressure boundary conditions have been employed for performance analysis purposes. Note that for the investigated cases, changes in the exit flow static pressure conditions only affected the flow split between over- and underflow but did not impact phase separation behavior within the cyclone. The latter is observed by comparing Cases 3 and 3a (for the Schiller-Naumann Drag Model) and Cases 13, 4 and 20 (for the Grace Drag Model); not illustrated here.

\subsection{Effect of turbulence model}

Influence of the employed turbulence model on analysis results has been investigated for three specific cases, that is, Case 21 (high flow, high loading and large bubble size), Case 27 (high flow, small loading and small bubble size) and Case 25 (analogous to Case 21 but with only $20 \%$ of the volumetric flow rate).

For Case 21, results from the BSL EARSM and the steady-state SST-CC Model compare reasonably well in air and liquid mass flow rate splits between over- and underflow, predicted inlet pressure and overall velocity field (see Figs 7 (left) and 3 (right)). In comparison, air separation from the underflow is predicted to be significantly higher when using the SSG RSM (not illustrated) and the time-averaged transient results from the SST-CC model. Both of those model results also show significantly higher inlet pressures and a vortex core, which is more aligned with the cyclone centerline (Fig. 7, right).

For Case 27 conditions, flowfield predictions from BSL EARSM and steady-state SST-CC model follow each other more closely than under Case 21 operating conditions. The same is also true for the predicted pressure drop. In comparison, results from the BSL RSM and steady-state SST-CC model results follow each other even closer. All three turbulence models predict negligible phase separation while the SSG model results indicate formation of a thin film in the conical section and at the underflow exit. Similar to Case 21 conditions, the pressure drop across the cyclone predicted by the SSG model and the transient SST-CC model run are significantly higher than predicted by the other models and with a vortex core more closely following the cyclone axis (see Fig. 8). 

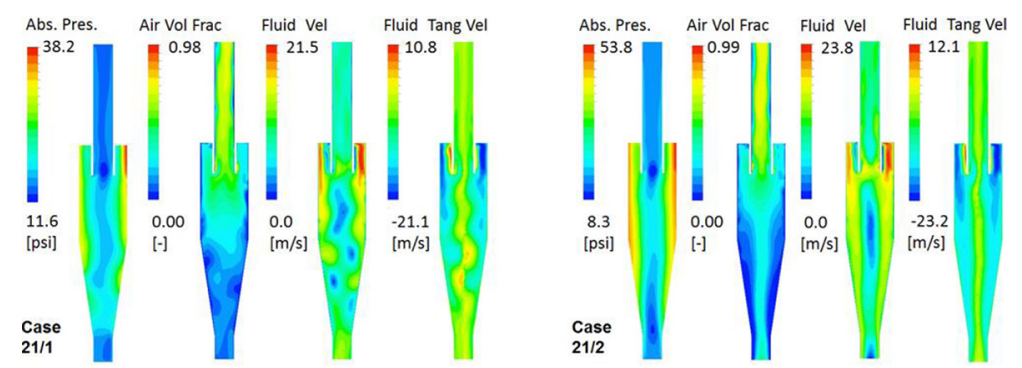

Figure 7: Contour plots of absolute static pressure, air volume fraction, fluid absolute velocity and fluid circumferential velocity for Case 21 operating conditions and with SST-CC model for steady-state run (left, 21/1) and time-averaged unsteady run (right, 21/2).
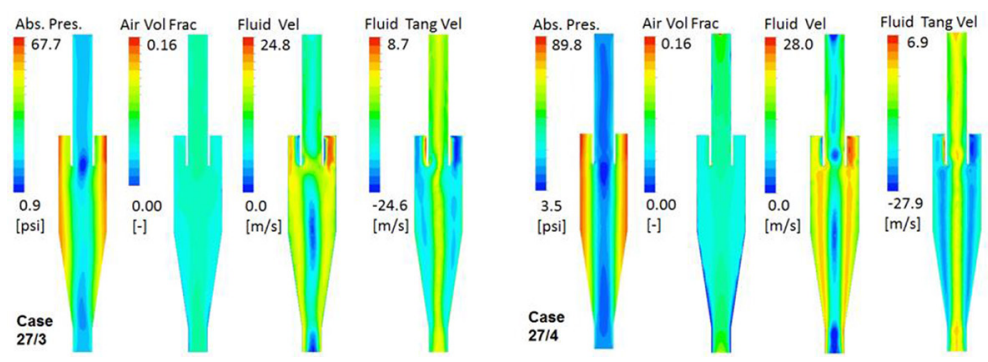

Figure 8: Contour plots of absolute static pressure, air volume fraction, fluid velocity and fluid circumferential velocity for Case 27 conditions from instantaneous transient result using SST-CC model (left, 27/3) and steady-state results based on SSG RSM (right, 27/4).

For Case 25 conditions, that is, high loading, large bubble size but low flow rate, results from the SSG RSM and the transient results from the SST-CC model match each other very well in pressure drop and air/liquid over- and underflow. In addition, both models predict a notable phase separation with about 50\% air volume fraction in the overflow even though the liquid mass flow through both over- and underflow is comparable (and without wall film formation in the overflow).

In contrast, the BSL EARSM model does not predict significant phase separation for this case. Discrepancies in predicted inlet pressure between the BSL EARSM and the two prescribed turbulence models are not as severe as found for the operating conditions of Cases 21 and 27 (see Fig. 9).

\section{SUMMARY}

A hydrocyclone for separating dispersed air bubbles in a liquid oil primary phase has been investigated numerically using ANSYS CFX. Analysis results differ depending on the employed turbulence model and the interphase particle drag model. Most notable differences were found at large bubble sizes and large flow rates. Unsteady results using the SST model with curvature correction (CC) and steady-state results using the SSG RSM compared generally well. However, differences between those results and steady-state results based on BSL EARSM or the SST-CC model were considerable at large flow rates. Nevertheless, the BSL 

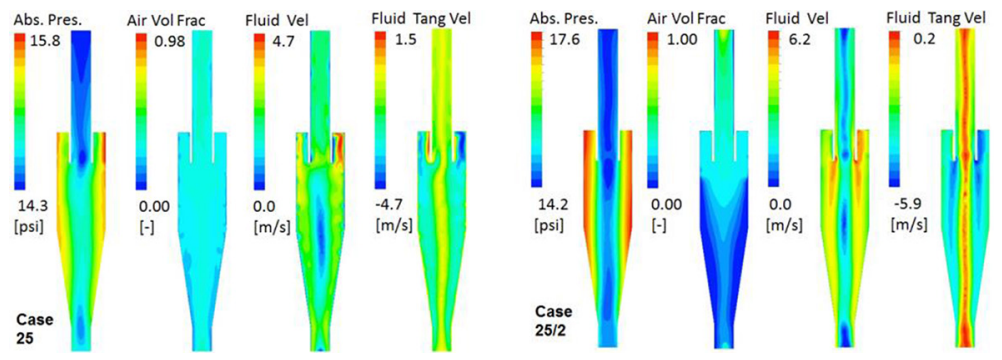

Figure 9: Contour plots of absolute static pressure, air volume fraction, fluid absolute velocity and fluid circumferential velocity for Case 25 using BSL EARSM (left) and corresponding case with SSG RSM turbulence model (right).

EARSM can be useful in making qualitative design choices, for example, in assessing bubble size separation limits as a function of Reynolds number and axial-to-tangential velocity ratios. The latter results (not illustrated in this paper) compared favorably with existing literature [16].

\section{REFERENCES}

[1] Swanbom, R.A., A new approach to the design of gas-liquid separators for the oil industry, Technical University Delft, Delft, The Netherlands, 1988.

[2] Slettebo, E.S., Separation of gas from liquids in viscous systems, NTNU Norwegian University of Sciences and Technology, Trondheim, Norway, 2009.

[3] Moore, J.Z.; Somoza, R.J.; Shih, A.J.; Filipi, Z.S; Moskalik, A.J.; Johnson, N.M., Characterization of the fluid deaeration device for a hydraulic hybrid vehicle system, SAE Technical Paper 2008-01-0308, 2008.

[4] Hoyt, N., et al., Cyclonic two-phase flow separator experimentation and simulation for use in a microgravity environment. Journal of Physics, 327, pp. 12-56, 2011. doi: http://dx.doi.org/10.1088/1742-6596/327/1/012056

[5] Kelsall, D.F., A study of the motion of solid particles in a hydraulic cyclone. Transactions of the Institution of Chemical Engineers, 30, p. 87, 1952.

[6] Bradley, D. \& Pulling, D.J., Flow patterns in the hydraulic cyclone and their interpretation in terms of performance. Transactions of the Institution of Chemical Engineers, 37(1959), p. 34, 1959.

[7] Bradley, D., The Hydrocyclone, Pergamon Press Limited: Oxford, 1965.

[8] Svarovsky, L., Hydrocyclones, Holt Rinehart and Winston: London, 1984.

[9] Hoffman, A.C. \& Stein, L.E., Gas Cyclones and Swirl Tubes: Principles, Design and Operation, Springer: Berlin, 2002.

[10] Cortes, C. \& Gil, A., Modeling the gas and particle flow inside cyclone separators. Progress in Energy and Combustion Science, 33, pp. 409-452, 2007. doi: http://dx.doi. org/10.1016/j.pecs.2007.02.001

[11] Boysan, F.; Ayers, W.H.; Swithenbank, J.A., A fundamental mathematical modeling approach to cyclone design. Transactions of the Institution of Chemical Engineers, 60, pp. 222-230, 1982.

[12] Pericleous, K. \& Rhodes, N., The hydrocyclone classifier - a numerical approach. International Journal of Mineral Processing, 17, pp. 23-43, 1986. doi: http://dx.doi. org/10.1016/0301-7516(86)90044-X 
[13] Hsieh, K. \& Rajamani, R., Mathematical model of the hydrocyclone based on physics of fluid flow. AIChE Journal, 37, pp. 735-746, 1991. doi: http://dx.doi.org/10.1002/ aic. 690370511

[14] Hsieh, K., Phenomenological model of the hydrocyclone, PhD Thesis, Department of Metallurgy and Metallurgical Engineering, University of Utah, 1988.

[15] Utikar, R.; Darmawan, N.; Tade, M.; Li, Q.; Evans, G.; Glenny, M.; Pareek, V., Hydrodynamic simulation of cyclone separator (Chapter 11). Computational Fluid Dynamics, ed. H.W. Oh, InTech: Croatia, pp. 241-266, 2010.

[16] Mantilla, I.; Shirazi, S.A.; Shoham, O., Flow field predictions and bubble trajectory model in gas-liquid cylindrical cyclone (GLCC) separators. ASME Journal, 121, pp. 9-15, 1999. doi: http://dx.doi.org/10.1115/1.2795063

[17] Menter, F., Two-equation eddy-viscosity turbulence models for engineering applications. AIAA Journal, 32(8), pp. 1598-1605, 1994. doi: http://dx.doi.org/10.2514/3.12149 